\title{
p53 and erbB-2 are not associated in matched cases of primary and metastatic ovarian carcinomas
}

\author{
Cristina Rodríguez-Burford ${ }^{\mathrm{a}}$, David C. Chhieng ${ }^{\mathrm{a}}$, Cecil R. Stockard ${ }^{\mathrm{a}}$, Marc J. Kleinberg ${ }^{\mathrm{b}}$, \\ Mack N. Barnes ${ }^{\mathrm{b}}$, Edward E. Partridge ${ }^{\mathrm{b}}$, Heidi L. Weiss ${ }^{\mathrm{c}}$ and William E. Grizzle ${ }^{\mathrm{a}, *}$ \\ ${ }^{\mathrm{a}}$ Department of Pathology, ${ }^{\mathrm{b}}$ Department of Obstetrics and Gynecology, and ${ }^{\mathrm{c}}$ Comprehensive Cancer Center \\ Biostatistics Unit, The University of Alabama at Birmingham, Birmingham, AL 35294, USA
}

\begin{abstract}
Ovarian cancer has a high mortality rate largely due to the limited number of ovarian carcinomas detected at an early stage. Understanding the molecular changes occurring during the progression of ovarian carcinoma would aid in the development of therapies that may inhibit or target metastasis. Primary and metastatic lesions from 54 and 40 patients with advanced ovarian carcinoma, respectively (including matched primary and metastatic lesions from 30 patients) were evaluated for nuclear accumulation of p53 (clone BP53-12-1) and cytoplasmic and membranous immunostaining of p185 erbB-2 (clone 3B5) by immunohistochemistry. No differences in the immunostaining of p53 and p185erbB-2 (cytoplasm or membrane) were observed between primary and metastatic lesions of the matched cases. Similarly, no differences in the proportion of positive cases of p53 between primary and metastatic lesions of the matched cases was observed. Thus, novel therapies that target p53 or p185erbB-2 can utilize specimens from either primary or metastatic lesions to characterize these targets prior to therapy. Spearman correlations between p53 and p185erbB-2 (cytoplasm or membrane) immunohistochemistry scores were insignificant for the matched cases, all primary lesions, and all metastatic lesions. Also, no significant associations occurred between nuclear accumulation of p53 (positive versus negative) and phenotypic expression of p185erbB-2 (cytoplasm or membrane) immunostaining scores for the matched cases, all primary lesions, and all metastatic lesions. Thus, the nuclear accumulation of p53 and immunostaining of p185erbB-2 in the cytoplasm or on the cellular membranes are independent.
\end{abstract}

Keywords: Ovarian carcinoma, molecular markers, p53, and p185 erbB-2

\section{Introduction}

Ovarian carcinoma remains as the leading cause of death among gynecologic malignancies in the Western World [8]. Among ovarian carcinomas, tumors hypothesized to arise from the surface epithelium of the ovary are the most common, consisting of $80-90 \%$ of cases [18]. Due to a lack of specific symptoms, the majority of ovarian carcinomas usually presents at an advanced stage and thus, lead to poor survival [13]. To

*Address for correspondence: W.E. Grizzle, M.D., Ph.D., Dept. of Pathology, The University of Alabama at Birmingham, AL 35294, USA. E-mail: grizzle@path.uab.edu. date, the molecular mechanisms of ovarian carcinoma are poorly understood. The prognosis of a patient is largely dependent on morphologic criteria. No tissue specific markers have been identified to determine the genetic etiology of ovarian cancer.

A primary objective of ovarian carcinogenesis studies has been to characterize biomarkers useful for prognosis of ovarian cancer. These biomarkers include oncogenes and tumor suppressor genes. The tumor suppressor gene, p53, is commonly mutated in cancer. Overexpression of p53 has been observed in $50 \%$ of lung, colon, and ovarian cancers [19]. Mutations in p53 have been described as a late genetic change in ovarian carcinogenesis $[16,17]$. The p185 erbB-2 (HER$2 /$ neu) proto-oncogene encodes a $185-\mathrm{kDa}$ transmem- 
brane protein that has sequence homology with epidermal growth factor receptor may also play an important role in ovarian carcinogenesis [20]. The oncogene product of p185 erbB-2 is expressed by most ovarian cancers and overexpressed in approximately 15-30\% of these cancers [7]. Overexpression of p185erbB-2 at the cellular membrane has been associated with poor survival [2,20,24].

Studies have reported overexpression of p185erbB-2 together with p53 nuclear accumulation in ovarian carcinomas $[4,7,12,26]$. Some of these studies have observed a difference between benign and malignant ovarian tumors such that no or low expression of p53 and p185 erbB-2 are observed in benign lesions compared with nuclear accumulation of p53 and overexpression of erbB-2 in malignant lesions of the ovary $[1,11,12$, 26]. However, a limited number of studies have been published focusing on the relationship between p53 and p185 erbB-2 in individual tumors $[2,3,5,6,25]$. Thus, we are interested in determining if any differences in expression of p53 and p185 erbB-2 are observed between primary and metastatic lesions of the ovary. Understanding the molecular changes occurring during ovarian carcinogenesis would aid in the development of targeted interventions that may inhibit or target metastasis. In this analysis, primary and metastatic cases, including matched cases of primary and metastatic lesions were evaluated for nuclear accumulation of p53 and cytoplasmic and membranous immunostaining of p185 erbB-2 in patients with advanced chemotherapy resistant ovarian carcinoma.

\section{Materials and methods}

Paraffin-embedded tissues of patients who underwent surgery for ovarian epithelial neoplasia were collected from hospitals throughout the Southeastern United States during 1993-1999 as part of therapeutic trials at the University of Alabama at Birmingham. Thus, our study included only patients with advanced ovarian carcinoma who ultimately developed peritoneal metastasis and failed conventional chemotherapy. All patients were treated with surgical debulking and platinum based chemotherapy from 1993 to 1999. A total of 54 primary and 40 metastatic cases, including 30 matched primary and metastatic cases, were collected.

\subsection{Immunohistochemistry}

Paraffin-embedded tissues were sectioned at $5 \mu \mathrm{m}$ and attached to SuperFrost/Plus slides (Fisher Scientific, Norcross, GA) by heating at $58^{\circ} \mathrm{C}$ for one hour. Immunohistochemistry was performed as previously described $[9,10,15]$. Tissue sections were deparaffinized in xylene (three changes, 5 minutes each) and subsequently rehydrated in sequential ethanols for 5 minutes with one change each of absolute, $95 \%$ ethanol, and $70 \%$ ethanol. The sections were then transferred to a Tris-buffer bath $(0.05 \mathrm{M}$ Tris base, $0.15 \mathrm{NaCl}$, $0.0002 \%$ Triton X-100, $\mathrm{pH} 7.6$ ).

Each section was treated with an aqueous solution of $3 \% \mathrm{H}_{2} \mathrm{O}_{2}$ for 5 minutes in order to quench endogenous peroxidase activity. Each section was then incubated with $1 \%$ goat serum at room temperature for 20 minutes in order to reduce non-specific immunostaining. A monoclonal antibody specific for p53 (clone BP53 12.1, Biogenex, San Ramon, CA) and a monoclonal antibody specific for p185 erbB-2 (clone, 3B5, Oncogene Science, Cambridge, MA) were used at dilutions/concentrations of 1:400 and $0.25 \mathrm{ug} / \mathrm{ml}$, respectively. Primary antibodies were incubated for $1 \mathrm{hr}$ at room temperature. Negative controls remained in goat serum $(1 \%)$ at room temperature for $1 \mathrm{hr}$.

Primary antibodies were detected using a multispecies detection system from Biogenex (San Ramon, CA) or subsequently Signet Laboratories, Inc. (Dedham, MA). The sections were exposed to an antimouse and anti-rabbit antibody for 20 minutes and a peroxidase-labeled streptavidin was added for $20 \mathrm{~min}$ utes. A diaminobenzidine tetrachloride (DAB) super sensitive substrate kit (Biogenex, San Ramon, CA) was used to visualize the antibody-antigen complex. Then each section was counterstained using hematoxylin, dehydrated using graded alcohols, and soaked in xylene prior to attaching coverslips with permount.

The slides were examined by at least two investigators (CR-B, DCC, and WEG), each of whom estimated the intensity and extent of immunostaining. For p53 and p185 erbB-2, tumor cells were classified with respect to percentage of cells stained at each intensity from 0 (no staining) to 4 (highest intensity staining) [9]. If the staining intensity was no greater than the negative controls, then a score of zero was assigned for a specimen. A cytoplasmic and membrane immunostaining score was determined for $\mathrm{p} 185$ erbB-2 whereas a nuclear immunostaining score was determined for $\mathrm{p} 53$. The percentage of nuclei stained positive for p53 was also evaluated and a percentage of $10 \%$ or greater was considered positive p53 staining. 


\subsection{Statistical analysis}

Statistical analyses were conducted on the matched cases. The Wilcoxon Signed- Rank test was used to test if there was a difference between p53 immunostaining scores in the primary lesions versus the metastatic lesions in the matched cases $(n=30)$. Similarly, the Wilcoxon Signed-Rank test was used to test if there was a difference between 185 erbB-2 (membrane and cytoplasm) primary immunostaining scores and p185 erbB2 (membrane and cytoplasm) metastatic immunostaining scores in the matched cases $(n=26)$. A Spearman correlation was utilized to determine if any associations between p53 and p185 erbB-2 (cytoplasm or membrane) immunostaining scores in the matched cases were observed. The association of p185 erbB-2 (cytoplasm or membrane) and $\mathrm{p} 53$ positivity (with positivity defined as $>10 \%$ ) between primary and metastatic lesions in the matched cases was determined using the Wilcoxon Two-Sample test. The McNemar test was used to test if a change in the percentage of p53 nuclear accumulation was significantly different between primary and metastatic lesions in the matched cases.

Statistical analyses were also conducted with all primary lesions. The primary lesions $(n=54)$ included primary lesions with matched metastatic lesions as well as primary lesions without matched metastatic lesions. A Spearman correlation was used to determine if any associations between p53 and p185 erbB-2 (cytoplasm or membrane) immunostaining scores were observed in all primary lesions. The Wilcoxon Two-Sample test was used to determine any associations between p53 positivity and erbB-2 (cytoplasm or membrane) immunostaining scores in all of the primary lesions. The same statistical analyses that were conducted on the primary lesions were performed with the immunohistochemistry scores for the metastatic lesions. The total number of metastatic lesions $(n=40)$ included metastatic lesions with matched primary lesions as well as metastatic lesions without matched primary lesions.

Descriptive statistics were determined for age, race, parity, menopausal status, hormone replacement therapy, stage, histological classification of tumors, and radiation treatment using SAS statistical software [23]. Kaplan-Meier survival curves were estimated for all patients overall as well as stratified by grade, stage, hormone replacement therapy treatment, and menopausal status. A percentage of nuclear accumulation of p53 of $10 \%$ or greater was considered positive. Immunohistochemistry scores of $\mathrm{p} 185$ erbB- 2 for the membrane and the cytoplasm that were above the median were consid- ered positive. Survival curves between $\mathrm{p} 53$ positive vs. p53 negative patients and between p185 erbB-2 positive vs. p185 erbB-2 negative patients were also estimated for all matched, primary and metastatic lesions. The log-rank test was used to determine if there were statistically significant differences in survival curves between the clinical variables and levels of the two molecular markers.

\section{Results}

Table 1 includes the descriptive statistics for grade, stage, and histologic subtype of ovarian carcinoma lesions included in this study. Overall, the majority of the patients had high grade (grade 3 ) and stage III ovarian carcinomas. The largest proportion of the ovarian carcinomas was of the serous histologic subtype. The average age of each patient was 57.4 and the average parity was 2.5. Sixty-six patients were Caucasian and 2 patients were black. Forty-three patients were of post-menopausal status, whereas 13 patients were of pre-menopausal status. Thirty-seven patients were not on hormone replacement therapy and 19 patients were receiving hormone replacement therapy.

A total of 54 primary and 40 metastatic lesions, including 30 primary and matched metastatic lesions were identified. Each lesion was evaluated for nuclear accumulation of p53 and cytoplasmic and membranous immunostaining of p185 erbB-2 (Fig. 1). For p53 evaluations, immunostaining scores as well as percentage positivity was determined. Only immunostaining scores were determined for $\mathrm{p} 185$ erbB-2.

For the matched cases, no significant differences in immunohistochemistry scores of p53 $(p=0.6018)$, p185 erbB-2 immunostaining in the cytoplasm ( $p=$ $0.6210)$, and p185 erbB-2 immunostaining in the membrane ( $p=0.7910$ ) were observed between primary and metastatic lesions. The mean differences and standard error means of the immunohistochemistry scores between primary and metastatic lesions of the matched cases for p185 erbB-2 membrane and p185 erbB-2 cytoplasm were $-0.07 \pm 0.11$, and $0.03 \pm 0.12$, respectively (Fig. 2). No difference was also observed with the proportion of positive cases of p53 between primary and metastatic lesions of the matched cases $(p=0.1797)$ (Table 2). Spearman correlations between p53 and p185 erbB-2 (cytoplasm and membrane) immunohistochemistry scores were insignificant for the matched cases, all primary lesions, and all metastatic lesions. Similarly, no significant associations occurred 
Table 1

Descriptive statistics for grade, stage, and histologic subtype of ovarian carcinomas

\begin{tabular}{lcc}
\hline Grade $^{\mathrm{a}}$ & Number of patients & Percentage of patients \\
\hline 1 & 5 & 9.8 \\
2 & 14 & 27.45 \\
3 & 32 & 62.75 \\
Tumor stage $^{\mathrm{b}}$ & Number of patients & Percentage of patients \\
I & 1 & 1.43 \\
IIB & 2 & 2.86 \\
IIC & 2 & 2.86 \\
III & 1 & 1.43 \\
IIIB & 5 & 7.14 \\
IIIC & 52 & 74.29 \\
IV & 7 & 10.00 \\
Histologic subtype & Number patients & Percentage of patients \\
Serous & 48 & 67.60 \\
Mucinous & 2 & 2.81 \\
Endometrial & 8 & 11.27 \\
Clear cell & 1 & 1.41 \\
Mixed histologic type & 3 & 4.23 \\
Adenocarcinoma NOS & 9 & 12.68 \\
\hline
\end{tabular}

${ }^{\text {a }}$ Data for grade of the ovarian carcinoma was unavailable for 20 patients.

${ }^{\mathrm{b}}$ Data for stage of the ovarian carcinoma was unavailable for 1 patient.

${ }^{\mathrm{c}}$ Adenocarcinoma not otherwise specified.

Table 2

Proportion of cases with nuclear accumulation of $\mathrm{p} 53\left(\mathrm{p} 53^{\mathrm{NAC}}\right)$ and negative for $\mathrm{p} 53\left(\mathrm{p} 53^{\mathrm{NEG}}\right)$ among the matched primary and metastatic lesions ${ }^{\mathrm{a}}$

\begin{tabular}{lc}
\hline Case description & Number of cases \\
\hline Primary Tumor + Metastatic Tumor, both p53 & 11 \\
Primary Tumor + Metastatic Tumor, both p53 & 14 \\
Primary Tumor p53NEG + Metastatic Tumor p53 & 1 \\
Primary Tumor p53NAC + Metastatic Tumor p53 & 4 \\
\hline
\end{tabular}

${ }^{\mathrm{a}} \mathrm{A} 10 \%$ or greater cut-off value was considered positive for $\mathrm{p} 53$.

between p53 positivity (positive versus negative) and erbB-2 (cytoplasm and membrane) immunohistochemistry scores for the matched cases, all primary lesions, and all metastatic lesions.

Primary lesions ( $n=45$ ) were evaluated for presence of adjacent uninvolved tissue. A total of 24 cases contained normal appearing uninvolved tissue. $\mathrm{Nu}-$ clear accumulation of p53 as well as cytoplasmic and membranous immunostaining of $\mathrm{p} 185$ erbB-2 was evaluated. The average p53 immunohistochemistry score ( $n=21$ ) was 0.0 . The average immunohistochemistry score for cytoplasmic and membranous expression of p185 erbB-2 was $0.71(n=19)$ and $0.095(n=19)$, respectively.

Survival estimates indicated that the median survival time was 36 months. Interestingly, there was no significant difference in survival time based on grade $(p=0.816)$, stage $(p=0.5181)$, menopausal status $(p=0.5433)$, and hormone replacement therapy treatment $(p=0.4566)$. Survival estimates based on the nuclear accumulation of p53 and the cytoplas- mic and membranous staining of p185 erbB-2 were also analyzed. Based on p53 positivity (positive versus negative), no significant differences were observed in survival time for primary lesions of matched cases $(p=0.2772)$, metastatic lesions of matched cases $(p=0.3460)$, all primary lesions $(p=0.7323)$, and all metastatic lesions ( $p=0.6012)$. For survival estimates of p185 erbB-2, immunohistochemistry scores of immunostaining in the membrane and of immunostaining in the cytoplasm that were above the median were considered positive. Based on the immunostaining of p185 erbB-2 in the membrane, no significant differences were observed with survival time for primary lesions of matched cases ( $p=0.7661)$, metastatic lesions of matched cases ( $p=0.7117)$, all primary lesions $(p=0.4631)$, and all metastatic lesions ( $p=0.5023)$. Similarly, no significant differences were found with immunostaining scores of erbB-2 in the cytoplasm and survival time for primary lesions of matched cases $(p=0.8589)$, metastatic lesions of matched cases 


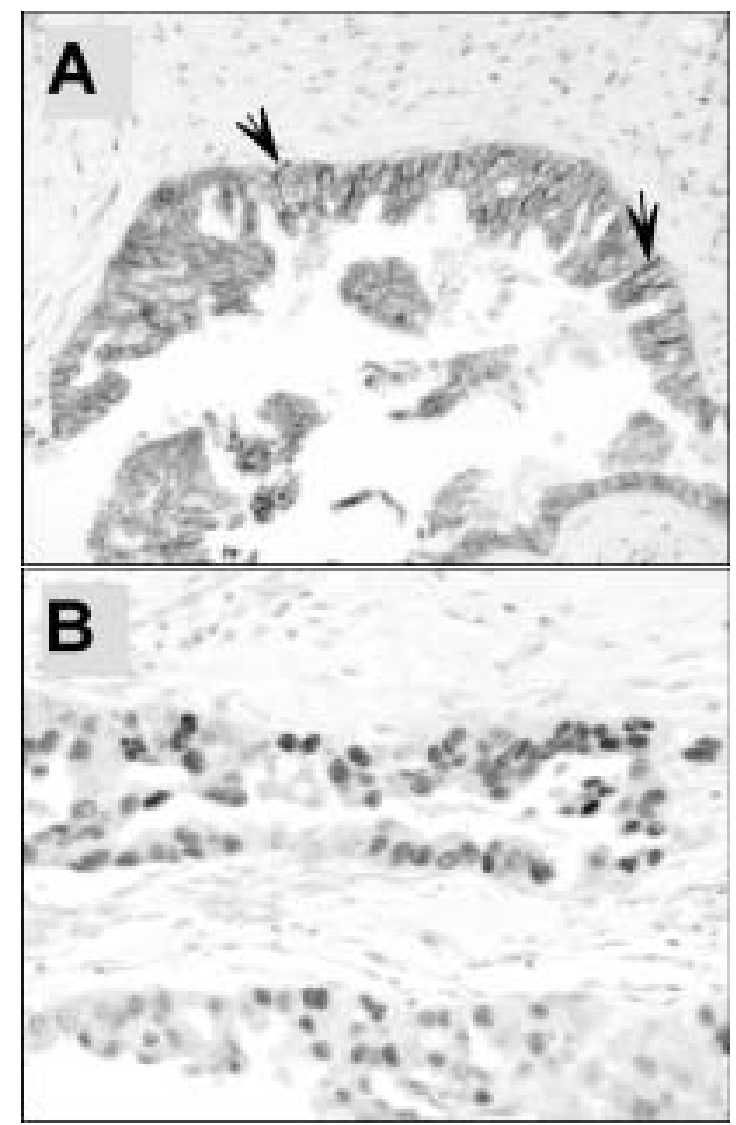

Fig. 1. Immunohistochemical staining of p185 erbB-2 on the cell surface membrane (solid arrow) and in the cytoplasm of some ovarian carcinoma cells (A). Immunohistochemical staining of p53 in the nucleus of some ovarian carcinoma cells (B).

$(p=0.5635)$, all primary lesions $(p=0.4066)$, and all metastatic lesions $(p=0.7364)$.

\section{Discussion}

The changes in expression of p185 erbB-2 and the nuclear accumulation of p53 have been studied in the progression of ovarian neoplasia. Several studies have determined differences between benign, borderline, and malignant lesions of the ovary with respect to nuclear accumulation of p53 and the phenotypic expression of p185 erbB-2. Anreder et al. [1] observed significantly elevated nuclear accumulation of $\mathrm{p} 53$ ( $p=0.001)$ and expression of $\mathrm{p} 185$ erbB-2 $(p=0.001)$ in ovarian carcinomas compared to borderline tumors. Harlozinska et al. [12] reported no evidence of p53 and p185 erbB-2 expression in benign neoplasms of the ovary, $30 \%$ p53 and 35\% p185 erbB-2 expression in borderline ovarian tumors, and $50 \%$ p53 and $48.5 \%$ p185 erbB-2 expression in ovarian carcinomas. Similarly, Harlozinska and Bar [11] observed no nuclear accumulation of p53 and expression of p185 erbB-2 in benign ovarian tumors and detected carcinomas, respectively. Mabrouk et al. [14] also observed an overexpression of p185 erbB-2 and mutant p53 proteins in malignant cases when compared with benign cases. Overexpression of p185 erbB-2 at the cellular membrane has been associated with poor survival in ovarian carcinogenesis [2,21,24]. Similarly, lack of nuclear accumulation of p53 or mutations in p53 have been associated with significantly greater disease-free survival than a high expression of p53 [22].

Most of the studies reporting differences between the expression of $\mathrm{p} 53$ and $\mathrm{p} 185$ erbB- 2 between benign and malignant lesions of the ovary, however, are not comparisons within the same tumor. Our findings of no differences in expression of p53 and p185 erbB-2 between primary and metachronous metastatic lesions in patients with advanced chemotherapy resistant ovarian carcinoma confirm the findings of a limited number of studies available for p53 and p185 erbB-2 comparisons within individual tumors. Daidone et al. [5] reported no differences in the nuclear accumulation of p53 between primary and synchronous metastatic sites in 61 women with ovarian carcinomas. Goff et al. [6] observed no significant changes in the nuclear accumulation of p53 and expression of p185 erbB-2 between primary and metachronous metastatic lesions in 23 women with advanced ovarian cancer. A larger study by Tewari et al. [25] also found no significant differences between the nuclear accumulation of p53 and expression of $\mathrm{p} 185$ erbB-2 in matched cases. Few of these studies reporting the expression of p185 erbB-2 between primary and metastatic lesions in individual ovarian tumors have reported immunostaining of p185 erbB-2 by cellular localization. To date, the cellular localization of p185 erbB-2 is not fully understood. Nevertheless, we observed immunostaining in the cytoplasm and in the membrane of tumor cells and did not find any significant differences between primary and metastatic lesions based on the cellular localization of p185 erbB-2.

Our study demonstrates that there are no differences in the nuclear accumulation of p53 and the phenotypic expression of p185 erbB-2 between primary and metastatic lesions (in patients with advanced chemotherapy resistant ovarian carcinoma) for the purposes of selecting tumors for novel therapies which target p53 and p185 erbB-2. Thus, when determining 



Fig. 2. Immunohistochemistry scores for p185 erbB-2 cytoplasmic (A) and membranous (B) staining among all primary and metastatic lesions for the matched cases $(n=26)$.

appropriate targets, a sample of a primary lesion may be just as useful as a sample of a metastastic lesion. However, future studies are necessary in determining if any changes in p53 and p185 erbB-2 are observed between early and late stage ovarian carcinomas.

\section{Acknowledgment}

Support provided by the Specialized Program of Research Excellence (SPORE) in Ovarian Cancer NCI \# CA83591-02. 


\section{References}

[1] M.B. Anreder, S.M. Freeman, A. Merogi, S. Halabi and A.J. Marrogi, p53, c-erbB-2, and PCNA status in benign, proliferative, and malignant ovarian surface epithelial neoplasms, Arch Pathol Lab Med 123 (1999), 310-316.

[2] A. Berchuck, A. Kamel, R. Whitaker, B. Kerns, A. Olt, R. Kinney, J.T. Soper, R. Dodge, D.L. Clarke-Pearson, P. Marks, S. MacKenzie, S. Yin and R.C. Bast Jr., Overexpression of Her$2 /$ neu is associated with poor survival in advanced epithelial ovarian cancer, Cancer Res 50 (1990), 4087-4091.

[3] A. Calugi, P. Eleuteri, D. Cavallo, N. Giuseppe, L. Albonici, M.P. Lombardi, V. Manzari, C. Romanini and R. DeVita, Detection of cellular heterogeneity by DNA ploidy, 17 chromosome, and p53 gene in primary carcinoma and metastasis in a case of ovarian cancer, Int J Gynecol Pathol 15 (1996), 77-81.

[4] K. Chang, I. Ding, F. Kern and M.C. Willingham, Immunohistochemical analysis of p53 and HER-2/neu proteins in human tumors, The Journal of Histochemistry and Cytochemistry 39(9) (1991), 1281-1287.

[5] M.G. Daidone, E. Benini, B. Valentinis, G. Tomasic, G. Bolis, A. Villa and R. Silvestrini, p53 expression, DNA content and cell proliferation in primary and synchronous metastatic lesions from ovarian surface epithelial - stromal tumours, Eur J Cancer 32A (1996), 1388-1393.

[6] B.A. Goff, J.A. Ries, L.P. Els, M.D. Coltrera and A.M. Gown, Immunophenotype of ovarian cancer as predictor of clinical outcome: evaluation at primary surgery and second-look procedure, Gynecol Oncol 70 (1998), 378-385.

[7] B.A. Goff, K. Shy, B.E. Greer, H.G. Muntz, M. Skelly and A.M. Gown, Overexpression and relationships of Her-2/neu, epidermal growth factor receptor, p53, Ki-67, and tumor necrosis factor $\alpha$ in epithelial ovarian cancer, J Gynaec. Oncol 17(6) (1996), 487-492.

[8] M. Green, J. Clark and D. Blayroy, The epidemiology of ovarian cancer, Semin Oncol 11 (1984), 209-226.

[9] W.E. Grizzle, R.B. Myers, U. Manne and S. Srivastava, Immunohistochemical evaluation of biomarkers in prostatic and colorectal neoplasia, in: John Walker's methods in molecular medicine-tumor marker protocols, M. Hanausek and Z. Walaszek, eds, Humana Press, Inc., Totowa, NJ, 1998, pp. 143-160

[10] W.E. Grizzle, D. Shibata, U. Manne, R.B. Myers and A.R. Frost, Molecular and histopathologic changes in the development of colorectal neoplasia, in: Molecular Pathology of Early Cancer, S. Srivastava et al., eds, IOS Press, Amsterdam, 1999, pp. $135-170$.

[11] A. Harlozinska and J.K. Bar, Relationship between p53 and cerbB-2 overexpression in tissue sections and cyst fluid cells of patients with ovarian cancer, Tumor Biol 15 (1994), 223-229.
[12] A. Harlozinska, J.K. Bar, E. Sobanska and M. Goluda, p53, c-erbB-2 and p21ras expression in tumor effusion cells of patients with histopathologically different ovarian neoplasms, Anticancer Research 17 (1997), 3545-3552.

[13] G.B. Kristensen and C. Tropé, Epithelial ovarian carcinoma, The Lancet 349 (1997), 113-117.

[14] G.M. Mabrouk, S.A.A. Sahar, K.I. El-Lamie and A. Khalifa, Quantitative measurement of c-erbB-2 p185 and mutant p53 expression in ovarian neoplasms by enzyme immunoassay, Clinical Chemistry 42 (1996), 981-982.

[15] U. Manne, R.B. Myers, C. Moron, R.B. Poczatek, S. Dillard, H. Weiss, D. Brown, S. Srivastava and W.E. Grizzle, Prognostic significance of Bcl-2 expression and p53 nuclear accumulation in colorectal adenocarcinoma, Int. J. Cancer 74 (1997), 346-358.

[16] R. Mazars, P. Pujol, T. Maudelone, P. Jeanteur and C. Theillet, p53 mutations in ovarian cancer: A late event? Oncogene 6 (1991), 1685-1690.

[17] C.H. Mok, S.W. Tsao, R.C. Knapp, P.M. Fishbaugh and C.C. Lau, Unifocal origin of advanced human epithelial ovarian cancers, Cancer Res 52 (1992), 5119-5122.

[18] R.P. Perez, A.K. Godwin and T.C. Hamilton, Ovarian Cancer Biology, Semin Oncol 18 (1991), 186-204.

[19] P.L. Porter, A.M. Gown, S.G. Kramp and M.D. Coltrera, Widespread p53 overexpression in human malignant tumors, Am J Pathol 140 (1992), 145.

[20] D.M. Reese and D.J. Slamon, Her-2/neu signal transduction in human breast and ovarian cancer, Stem Cells 15 (1997), 1-8.

[21] D.M. Reese and D.J. Slamon, HER-2/neu signal transduction in human breast and ovarian cancer, Stem Cells 15 (1997), $1-8$.

[22] K. Sakai, T. Kaku, T. Kamura, N. Kinukawa, S. Amada, T. Shigematsu, T. Hirakawa, H. Kobayashi, Ariyoshik and H. Nakano, Comparison of p53, Ki-67, and CD44v6 expression between primary and matched metastatic lesions in ovarian cancer, Gynecologic Oncology 72 (1999), 360-366.

[23] SAS Institute, Inc. SAS/STAT User's Guide. Version 6.0. Cary, NC: SAS Institute, Inc., 1990.

[24] D.J. Slamon, W. Godolphin, L.A. Jones, J.A. Holt, S.G. Wong and D.E. Keith et al., Studies of the HER-2/neu protooncogene in human breast and ovarian cancer, Science $\mathbf{2 4 4}$ (1989), 707-712.

[25] K.S. Tewari, A.S. Kyshtoobayeva, R.S. Mehta, I.-R. Yu, R.A. Burger, P.J. DiSaia and J.P. Fruehauf, Biomarker conservation in primary and metastatic epithelial ovarian cancer, Gynecologic Oncology 78 (2000), 130-136.

[26] C. van-Haaften-Day, P. Russell, C.M. Boyer, B.-J.M. Kerns, J.R. Wiener, D.N. Jensen, R.C. Bast Jr. and N.F. Hacker, Expression of cell regulatory proteins in ovarian borderline tumors, Cancer 77 (1996), 2092-2098. 


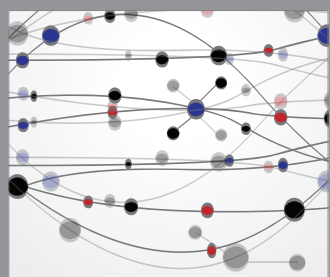

The Scientific World Journal
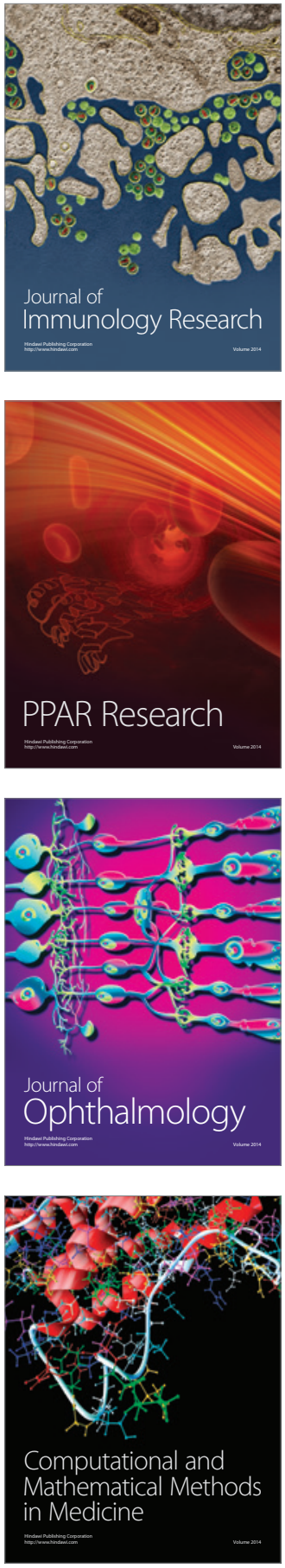

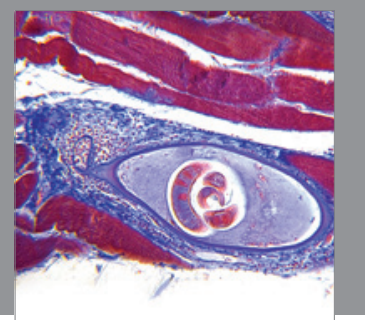

Gastroenterology

Research and Practice
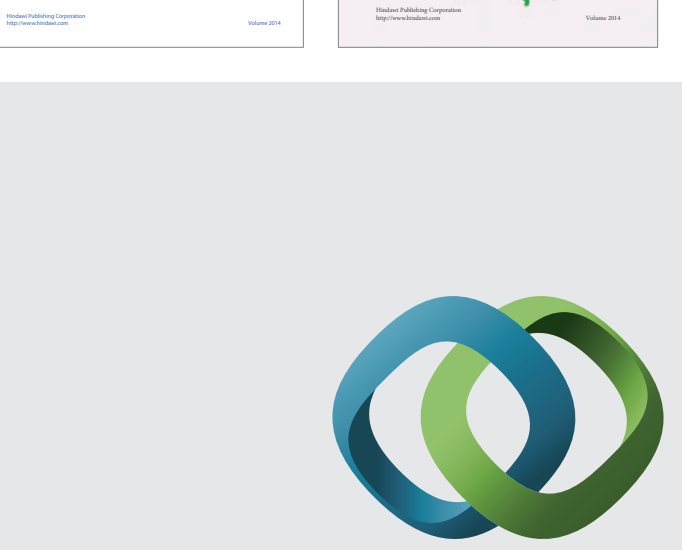

\section{Hindawi}

Submit your manuscripts at

http://www.hindawi.com
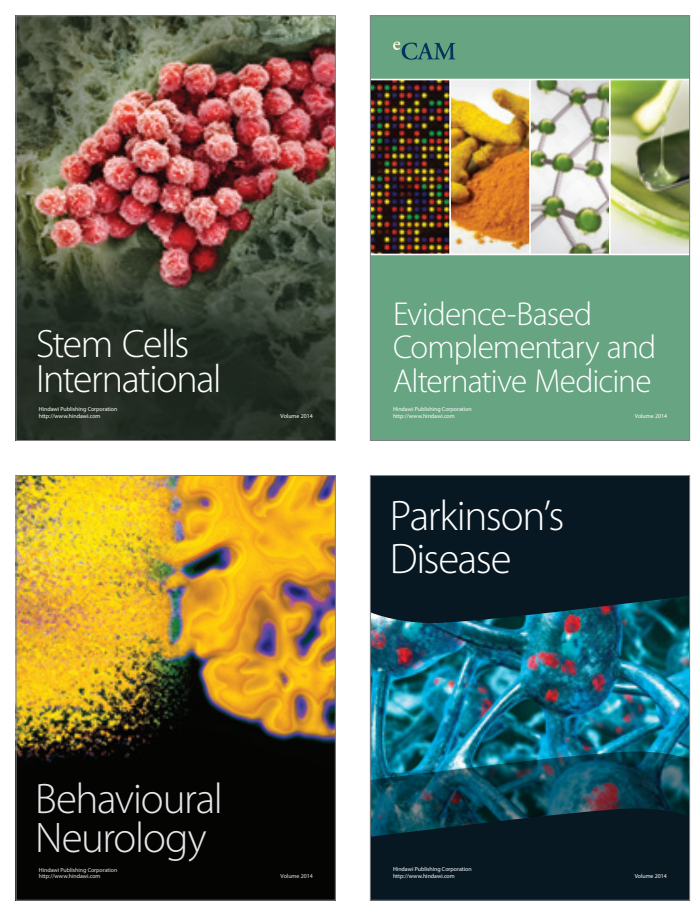

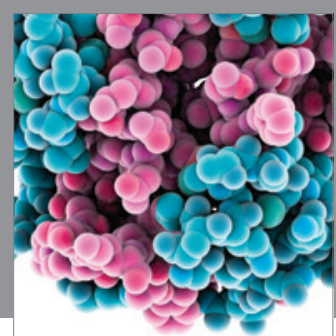

Journal of
Diabetes Research

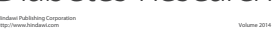

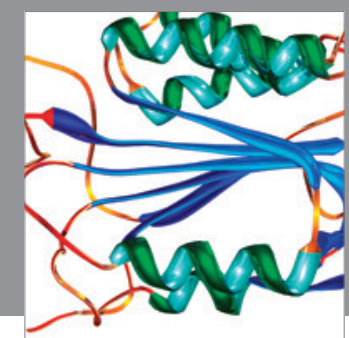

Disease Markers
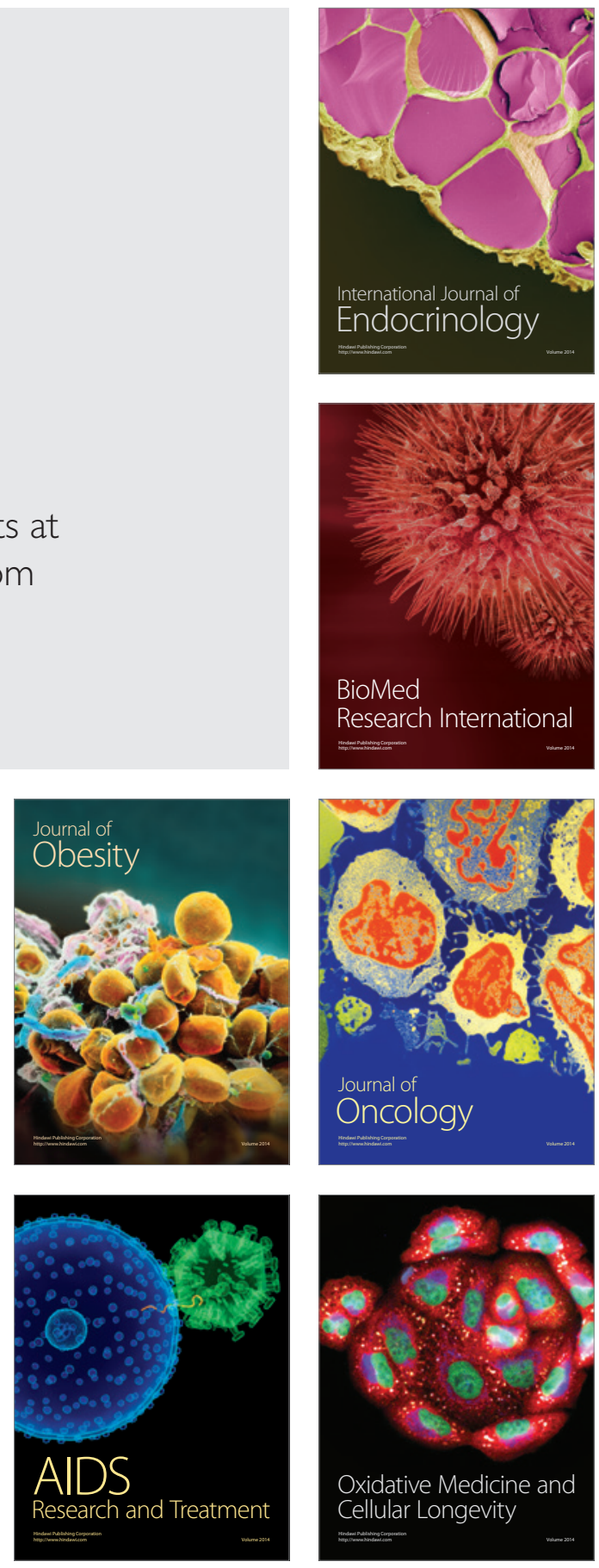